\title{
Heart Rate Variability Applied to Short-Term Cardiovascular Event Risk Assessment
}

\author{
Simao Paredes ${ }^{1}$, Teresa Rocha ${ }^{1}$, Paulo de Carvalho ${ }^{2}$, Jorge Henriques ${ }^{2}$, \\ Ramona Cabiddu ${ }^{3}$, João Morais ${ }^{4}$ \\ ${ }^{1}$ Computer Science and Systems Engineering Department, Polytechnic Institute of Coimbra (IPC/ISEC), Coimbra, Portugal \\ ${ }^{2}$ Centre for Informatics and Systems of the University of Coimbra, \\ Department of Informatics Engineering, University of Coimbra, Coimbra, Portugal \\ ${ }^{3}$ Dipartimento di Elettronica, Informazione e Bioingegneria, Politecnico di Milano, Milano, Italy \\ ${ }^{4}$ CardiologyDepartment, Leiria-Pombal Hospital Centre, Leiria, Portugal \\ Email: sparedes@isec.pt, teresa@isec.pt, carvalho@dei.uc.pt, jh@dei.uc.pt, \\ ramona.cabiddu@gmail.com, joaomorais@hsaleiria.min-saude.pt
}

Received June 2013

\begin{abstract}
Cardiovascular disease (CVD) risk assessment is an important instrument to enhance the clinical decision in the daily practice as well as to improve the preventive health care promoting the transfer from the hospital to patient's home. Due to its importance, clinical guidelines recommend the use of risk scores to predict the risk of a cardiovascular disease event. Therefore, there are several well known risk assessment tools, unfortunately they present some limitations. This work addresses this problem with two different methodologies: 1) combination of risk assessment tools based on fusion of Bayesian classifiers complemented with genetic algorithm optimization; 2) personalization of risk assessment through the creation of groups of patients that maximize the performance of each risk assessment tool. This last approach is implemented based on subtractive clustering applied to a reduced-dimension space. Both methodologies were developed to short-term CVD risk prediction for patients with Acute Coronary Syndromes without ST segment elevation (ACS-NSTEMI). Two different real patients' datasets were considered to validate the developed strategies: 1) Santa Cruz Hospital, Portugal, N = 460 patients; 2) Leiria-Pombal Hospital Centre, Portugal, N = 99 patients. This work improved the performance in relation to current risk assessment tools reaching maximum values of sensitivity, specificity and geometric mean of, respectively, 80.0\%, 82.9\%, 81.5\%. Besides this enhancement, the proposed methodologies allow the incorporation of new risk factors, deal with missing risk factors and avoid the selection of a single tool to be applied in the daily clinical practice. In spite of these achievements, the CVD risk assessment (patient stratification) should be improved. The incorporation of new risk factors recognized as clinically significant, namely parameters derived from heart rate variability (HRV), is introduced in this work. HRV is a strong and independent predictor of mortality in patients following acute myocardial infarction. The impact of HRV parameters in the characterization of coronary artery disease (CAD) patients will be conducted during hospitalization of these patients in the Leiria-Pombal Hospital Centre (LPHC).
\end{abstract}

Keywords: CVD Risk Assessment; Knowledge Management; Management of Cardiovascular Diseases; Decision-Support Systems

\section{Introduction}

Coronary heart disease (CHD) ${ }^{1}$, approximately half of all cardiovascular disease (CVD) deaths, is the single most common cause of death in Europe [1].

European Heart Network supports that around $80 \%$ of CHD are preventable [2], which shows that the improve-

\footnotetext{
${ }^{1}$ Coronary heart disease (heart attacks), cerebrovascular disease (stroke) raised blood pressure (hypertension), peripheral artery disease, rheumatic heart disease, congenital heart disease and heart failure are disorders of the heart and blood vessels globally designated by cardiovascular diseases (CVD).
}

ment of preventive health care can originate important benefits reducing the incidence of cardiovascular diseases.

Therefore, preventive health care assumes a critical importance in the present health care context. It is the key aspect in reducing the social and economic costs directly originated by cardiovascular diseases. In fact, it is commonly accepted that current health care paradigm has to move from reactive care towards preventive care, reducing the amount of in hospital care. Health telemonitoring systems are essential to achieve this target, as they allow the remote monitoring of patients who are in 
different locations away from the health care provider [3]. This remote monitoring is more challenging to the care provider, as the reliability/quality of the clinical decision must be guaranteed in order to optimize therapy.

The CVD risk assessment, i.e., the evaluation of the probability of occurrence of an event (death, myocardial infarction, hospitalization, disease development, etc.) give the patient's past and current exposure to risk factors, assumes great importance in this remote health monitoring. It contributes in providing the patient's health development as well as generating alarms [3]. In this way, a correct CVD risk assessment helps clinical professionals to identify the best treatment to each patient as well as to motivate the patient increasing the treatment compliance with the corresponding health benefits (patient seen as a co-producer of health) [4].

Additionally, clinical guidelines recommend the use of risk scores in the daily clinical practice to predict the risk of a cardiovascular disease event [5]. This assessment contributes in helping medical professionals in managing the patient population. Actually, physicians gather more information to identify the patients that need urgent hospitalization, those that need urgent review of respective care plans (lack of treatment, over treatment situations...) and those that correspond with the expected condition.

As a result, it is clinically recognized that the research and development of practical and accurate CV risk assessment tools ${ }^{2}$ are of vital importance [6]. In this context, several risk assessment tools were developed to assess the probability of occurrence of a CVD event within a certain period of time. Two types of risk may be calculated: absolute risk, i.e., probability of developing a CVD event over a given period of time (e.g. 10 years), and a relative risk, i.e., risk of someone developing a CVD event that has risk factors compared to an individual of the same age and sex who does not. Moreover, available risk assessment tools differ on the assessed period of time (short-term [months]/long-term [years]), predicted events (death/non-fatal), disease (coronary artery disease, heart failure, etc.), risk factors, patient condition (ambulatory patients, hospitalized patients, cardiac transplant candidates, etc.) [7].

In spite of their relevance, these risk assessment tools exhibit important drawbacks: 1) may present some lack of performance; 2) ignore the information provided by other risk assessment tools that were previously developed; 3) consider (each individual tool) a limited number of risk factors; 4) have difficulty in coping with missing risk factors; 5) do not allow the incorporation of additional clinical knowledge; 6) do not assure the clinical inter- pretability of the respective parameters; 7) impose a selection of a standard tool to be applied in the clinical

\footnotetext{
${ }^{2}$ In order to clarify, risk assessment models that have been statistically validated and are available in literature are going to be designated through this work as risk assessment tools.
}

practice.

This work addresses the identified weaknesses with two different methodologies: 1) combination of risk assessment tools (fusion of naïve Bayes classifiers complemented with genetic algorithm optimization); 2) personalization of risk assessment (creation of groups of patients based on subtractive clustering applied to a reduced-dimension space).

Two main hypothesis support the first approach: 1) it is possible to create a common representation of individual risk assessment tools; and 2) it is possible to combine individual models. The main goal is to integrate several sources of information (risk assessment tools) to defeat the identified limitations. Though, current risk assessment tools are diversely represented [8-10], which does not facilitate their integration/combination. Therefore a common representation must be created verifying some requirements: 1) simplicity; 2) ability to incorporate new risk factors (empirical clinical knowledge); 3) clinical interpretability; and 4) ability to deal with missing risk factors. The creation of a flexible framework based on the combination of available knowledge, is the basis of the second hypothesis. According to various authors an ensemble of classifiers is often more accurate than any of the respective single classifiers. Thus, there are several methods to implement model's combination which can be organized in two main categories: 1) model output combination; and 2) model parameter/data fusion. The former includes the voting (e.g., voting, weighted voting, dynamic voting, bagging algorithms, boosting algorithms, etc.) and selection methods (e.g. information criteria, cross-validation variants, dynamic selection, etc.) [11,12]. Model parameter/data fusion implements a direct combination of the parameters of individual models $[13,14]$. The approach proposed in this work is included in this last category and explores the particular features of Bayesian inference mechanism. This framework also permits the implementation of optimization methodologies to increase the CVD risk prediction performance.

The second methodology, personalization of risk assessment, addresses the problem of the low performance exhibited by the current risk assessment tools when applied to the general population. The methodology is based on the evidence that risk assessment tools perform differently among different populations. Thus, the main hypothesis that supports this methodology can be stated as: if the patients are properly grouped (clustered) it would be possible to find the best classifier for each group.

The two methodologies were applied to three (GRACE [10], TIMI [8], PURSUIT [9]) well-accepted risk assessment tools [15]. The validation phase was supported by two real ACS-NSTEMI patient testing datasets: $i$ ) Santa Cruz Hospital, Lisbon/Portugal, N = 460 patients; 
ii) LeiriaPombal Hospital Centre, Portugal, $\mathrm{N}=99$.

In spite of these achievements, the CVD risk assessment (patient stratification) should be improved. The possible incorporation of new risk factors recognized as clinically significant, namely parameters derived from heart rate variability (HRV), is introduced in this work. In fact, HRV is a strong and independent predictor of mortality in patients following acute myocardial infarction.

The paper is organized as follows: section II presents the developed methodologies. In section III some results of the validation procedure are discussed. Section IV depicts the incorporation of the heart rate variability parameters in the CVD risk assessment. The main conclusions are derived.

\section{Methodology}

Figure 1 presents the developed strategies. These methodologies were further detailed in previous publications of this research team $[16,17]$.

\subsection{Combination of Individual Tools}

The implementation of this approach is composed of two main phases: 1) common representation of individual risk assessment tools based on naïve Bayes classifier; 2) a combination scheme that exploits the probabilistic nature of naïve-Bayes inference mechanism complemented with an optimization based on genetic algorithms (GA).

\subsubsection{Common Representation of Individual Tools}

Current individual risk scores (risk assessment tools) are diversely represented (equations/scores/charts) which hinder their combination. To allow the fusion (combination) of these risk scores a common representation is created. The classifier selected to implement this common representation is the naïve Bayes classifier as it presents some important features: 1) simplicity; 2) ability to deal with missing risk factors; and 3) interpretability [18]. Its inference mechanism assumes that observations (attributes) are conditionally independent, given the value of hypothesis C:

$$
P(C \mid \mathbf{x})=P\left(C \mid X_{1}, \ldots, X_{p}\right)=\alpha P(C) \prod_{i=1}^{p} P\left(X_{i} \mid C\right)
$$

The term $P(C \mid \mathbf{x})$ is the probability that the hypothesis is correct (e.g., the risk is high) given a set of attributes $\mathbf{x}=\left[X_{1}, \ldots, X_{p}\right]$ (e.g., demographic data, clinical examination, laboratory measurements, etc.). $P(C)$ gives the prevalence of each risk level (a priori probability) and $P(X \mid C)$ expresses the probability of the observation $X$ given the value of class of risk $C$ (likelihood), $\alpha$ is a normalization constant.

The process of representing a specific individual risk

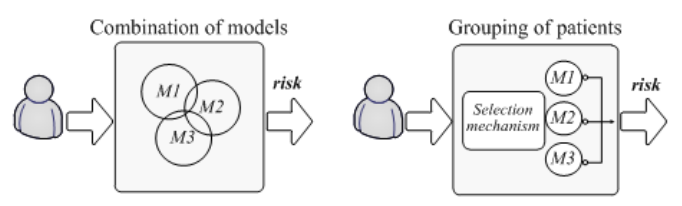

Figure 1. Proposed methodologies.

assessment tool as a naïve Bayes classifier can be systematized as follows: 1 ) a training datasetis generated, $N$ instances $\mathbf{x}=\left[X_{1}, \ldots, X_{p}\right]$ composed of $p$ attributes; 2) each instanceis applied to the risk assessment tool in order to obtain a complete labeled dataset

$J=\left\{\left(\mathbf{x}_{1}, c_{1}\right), \ldots .,\left(\mathbf{x}_{N}, c_{N}\right)\right\}$; and 3$)$ based on $J$ and through the maximum likelihood estimation method, the naïve Bayes classifier that resembles the behavior of that specific risk assessment tool is derived [16]. The probability $P(C)$ results directly from distribution of the class values (low risk/high risk patients).

\subsubsection{Individual Models Parameters’ Weighted Average}

The Equation (2) implements the proposed combination scheme, where it is possible to assign different weights for the individual Bayesian models.

$$
\begin{aligned}
& P(C)=\sum_{j=1}^{l} P\left(C_{j}\right) \times \frac{w_{j}}{\Gamma} \quad \text { wh еe } \Gamma=\sum_{j=1}^{l} w_{j} \\
& P\left(X_{i} \mid C\right)=\sum_{j=1}^{b} P\left(X_{i}^{j} \mid C_{j}\right) \times \frac{w_{j}}{\vartheta} \text { wh re } \vartheta=\sum_{j=1}^{b} w_{j}
\end{aligned}
$$

Value $l$ is the number of individual models, $b$ is the number of individual models that contain the attribute $X_{i}, C_{j}$ denotes each individual model, $w_{j}$ is the weight of model $j$.

An optimization based on GA can be performed. The GAfocuses on the $P\left(X_{i} \mid C\right), P(C)$ that are the parameters of the global model originated through the combination method. The optimization is performed in the neighbourhood of the initial values and through a multi-objective approach where sensitivity and specificity should be maximized. A detailed approach to this optimization procedure can be found on [16].

\subsection{Personalization Based on Grouping of Patients}

This second methodology was developed to enhance the performance of the risk prediction when compared to the one obtained with current risk assessment tools. It is based on the hypothesis that it is possible to select the most appropriate current risk assessment tool for a specific group of patients.

This methodology is composed of two main phases: 1) grouping of patients; and 2) identification of risk tools.

Grouping of patients is supported on a dimension re- 
duction step as it facilitates clustering; it avoids the heterogeneity (continuous, Boolean, etc.) of risk factors and it assures the uniformization of each patient's data (same scale). A non-linear mapping is implemented directly supported on the outputs of the selected set of risk assessment tools [17]. Thus, all instances

$\mathbf{X}_{i}=\left[x_{1}^{i} \ldots X_{P}^{i}\right]^{T} \in \mathbf{X}_{P \times N}$, that correspond to the $N$ patients are mapped into $\mathbf{y}_{i} \in \mathbf{Y}_{Q \times N}, i=1, \ldots, N$ where $y_{q}^{i}$ denotes the output of tool $q$ to classify the patient $i$. Then, clustering is applied through subtractive clustering [19]. Patients are grouped, based on the outputs of the risk tools $\mathbf{Y}_{Q \times N}$, in order to create $K$ disjoint groups (clusters) of patients with similar characteristics.

The second phase is the identification of risk assessment tools, where the performance of the several individual tools is assessed within each cluster. This allows that each cluster be assigned to the tool that presents the best performance. The final classification of a particular patient that belongs to a given cluster corresponds to the classification obtained with the individual tool that has the best performance with patients from that cluster [17].

\section{Results}

The two developed methodologies were applied to coronary artery disease patients (secondary prevention/short term) (Table 1).

The three risk assessment tools (TIMI [8], PURSUIT [9], GRACE [10]) were selected as they are the most well accepted/known CVD risk assessment tools specific for CAD patients [15].

Two testing ACS-NSTEMI real patient datasets were applied in the validation procedure: 1) Santa Cruz hospital with $\mathrm{N}=460$ patients. The event rate of combined endpoint (death/myocardial infarction) is $7.2 \%$. 2) LeiriaPombal Hospital Centre with $\mathrm{N}=99$ patients with an endpoint rate of $5.1 \%$.

The training dataset was created $\mathbf{x}^{i}=\left[x_{1}^{i} \ldots x_{p}^{i}\right]$ for all $i$; $1 \leq i \leq N$, with $N=1000$, based on the approach proposed in [14].

Table 1. Short-term risk assessment models.

\begin{tabular}{|c|c|c|c|c|}
\hline Model & Event & Time & Prev. & Risk Factors \\
\hline GRACE [10] & $\begin{array}{l}\mathrm{D} \\
\mathrm{MI}\end{array}$ & $6 \mathrm{~m}$ & Sec. & $\begin{array}{l}\text { Age, SBP, CAA HR, Cr, } \\
\text { STD, ECM, CHF }\end{array}$ \\
\hline PURSUIT [9] & $\begin{array}{l}\text { D } \\
\text { MI }\end{array}$ & $30 \mathrm{~d}$ & Sec. & $\begin{array}{l}\text { Age, Sex, SBP, CCS, HR, } \\
\text { STD, ERL, HF }\end{array}$ \\
\hline TIMI [8] & $\begin{array}{c}\text { D } \\
\text { MI } \\
\text { UR }\end{array}$ & $14 \mathrm{~d}$ & Sec. & $\begin{array}{l}\text { Age, STD, ECM, KCAD, } \\
\text { AS, AG, RF }\end{array}$ \\
\hline
\end{tabular}

D: Death; MI: Myocardial Infarction; UR: Urgent revasc.; m: months; d: days; S: Secondary Prevention; $\mathbf{C r}$-Creatinine, HR-Heart Rate, CAACardiac Arrest at Admission, CHF-Congestive Heart Failure, STD - ST Segment. Depression, ECE_Elevated Cardiac Markers/Enzymes, KCADKnown CAD, ERL-Enrolment (MI/UA), HF-Heart Failure, CCSAngina classification, AS-Use of aspirin in the previous 7 days, AG-2 or more angina events in past $24 \mathrm{hrs}, \mathbf{R F}-3$ or more cardiac risk factors.

\subsection{Combination of Individual Tools}

Table 2 contains the comparison of the Bayesian global model with the individual risk assessment tools as well as with the voting model (based on the outputs of the three individual risk assessment tools).

Table 3 presents the results obtained after the optimization procedure based on GA operation.

Considering the obtained results in table, the optimization improved the capability of the global model to predict the risk. However, there were some test cases where the combination methodology did not achieve an improvement of the performance, namely of the specificity value.

\subsection{Personalization Based on Grouping of Patients}

This methodology was applied to the Santa Cruz hospital dataset (combined endpoint, D/MI), based on the same risk tools (TIMI [8], PURSUIT [9], GRACE [10]). The first step was the dimensionality reduction from the original $P=16$ risk factors to $Q=3$ outputs of the risk tools. The clusters were created and the performance of each tool in each cluster was assessed.

This strategy achieved a higher sensitivity than all the individual tools (the best individual sensitivity is $60.8 \%$ while the sensitivity for the proposed strategy is $72.9 \%$ ) (Table 4). It did not reduce the specificity, which shows the potential of this approach to improve the risk prediction.

More detailed results obtained with the validation of these two methodologies, can be found on [16,17].

\section{Final Considerations}

\subsection{Ongoing Research}

In spite of the performance enhancements, there are some research directions that must be pursued to improve the CVD risk assessment. The fusion of the two developed methodologies must be further explored (personalization). Furthermore, the flexibility of the combination methodology (Bayesian global model) allows the incorporation of parameters recognized as clinically significant to improve risk assessment, namely the heart rate variability (HRV).

\section{Heart Rate Variability}

Heart rate variability is an ECG derived signal consisting in the oscillation in the interval between consecutive heart beats [20].

Cardiac rhythmical activity is controlled by the autonomic nervous system (ANS) where the sympathetic system (arousal/activation) and parasympathetic system (inhibition) are the key elements. A significant correla- 
Table 2. Performances comparison-Santa cruz, (D/MI).

\begin{tabular}{|c|c|c|c|c|c|c|}
\hline & $\%$ & GRACE & PURSUIT & TIMI & ByG & Vot \\
\hline \multirow{3}{*}{ Orig. } & SE & 60.6 & 42.4 & 33.3 & 60.6 & 48.5 \\
\hline & SP & 74.9 & 74.2 & 73.5 & 67.0 & 75.6 \\
\hline & $G_{\text {mean }}$ & 67.3 & 56.0 & 49.4 & 63.4 & 60.6 \\
\hline \multirow{3}{*}{$\begin{array}{c}\text { Boot } \\
\text { samples } \\
\mathrm{n}=1000\end{array}$} & SE & $60.8(60.2 ; 61.3)$ & 42.4 (41.9; 43.1) & 33.5 (33.0; 34.0) & $60.6(60.1 ; 61.3)$ & $48.6(48.0 ; 49.2)$ \\
\hline & SP & $74.9(74.8 ; 75.1)$ & $74.2(74.1 ; 74.3)$ & 73.6 (73.5; 73.7) & $67.0(66.9 ; 67.2)$ & $75.6(75.5 ; 75.8)$ \\
\hline & $\mathrm{G}_{\text {mean }}$ & $67.3(67.0 ; 67.6)$ & 55.8 (55.5; 56.2) & 49.3 (48.9; 49.7) & $63.6(63.3 ; 63.9)$ & $60.3(60.0 ; 60.7)$ \\
\hline
\end{tabular}

SE: Sensitivity; SP: Specificity; D: Death; MI: Myocardial Infarction; (-;-) = 95\% CI; ByG—Bayesian Global Model, Vot—Voting.

Table 3. Performances comparison.

\begin{tabular}{|c|c|c|c|c|c|c|c|}
\hline & & \multicolumn{2}{|c|}{ Santa Cruz 30 days/D/MI } & \multicolumn{2}{|c|}{ Santa Cruz 30 days/D } & \multicolumn{2}{|c|}{ Santo André 30 days/D } \\
\hline & & ByG & ByGAO & ByG & ByGAO & ByG & ByGAO \\
\hline \multirow{3}{*}{ Orig. } & SE & 60.6 & 72.7 & 61.5 & 76.9 & 80.0 & 80.0 \\
\hline & SP & 67.0 & 69.1 & 65.7 & 70.7 & 67.0 & 82.9 \\
\hline & $G_{\text {mean }}$ & 63.4 & 70.9 & 63.5 & 73.7 & 73.2 & 81.5 \\
\hline \multirow{3}{*}{$\begin{array}{c}\text { Boot } \\
\text { Samples } \\
n=1000\end{array}$} & SE & $60.6(60.1 ; 61.3)$ & $72.9(72.4 ; 73.4)$ & $61.6(60.7 ; 62.5)$ & $77.3(76.5 ; 78.0)$ & $80.3(78.9 ; 81.5)$ & 79.8 (78.6; 81.0) \\
\hline & SP & $67.0(66.9 ; 67.2)$ & $69.1(69.0 ; 69.2)$ & $65.8(65.6 ; 65.9)$ & $70.6(70.5,70.8)$ & $66.8(66.4 ; 67.2)$ & $83.8(83.3 ; 84.2)$ \\
\hline & $G_{\text {mean }}$ & $63.6(63.3 ; 63.9)$ & 70.9 (70.6; 71.1) & $63.1(62.7 ; 63.6)$ & $73.6(73.3 ; 74.0)$ & $72.3(71.5 ; 73.1)$ & $80.9(80.0 ; 81.6)$ \\
\hline
\end{tabular}

ByG_-Bayesian Global Model; ByG AO_Bayesian Global Model after Optimization.

Table 4. Performances comparison—Santa cruz (Death/MI).

\begin{tabular}{cccccc}
\hline & $\%$ & GRACE & PURSUIT & TIMI & Groups \\
\hline $\begin{array}{c}\text { Boot. } \\
\text { samples }\end{array}$ & SE & $60.8(60.2 ; 61.3)$ & $42.4(41.9 ; 43.1)$ & $33.5(33.0 ; 34.0)$ & $72.9(72.6 ; 73.5)$ \\
$\mathrm{n}=1000$ & SP & $74.9(74.8 ; 75.1)$ & $74.2(74.1 ; 74.3)$ & $73.6(73.5 ; 73.7)$ & $74.9(74.8 ; 75.1)$ \\
\hline
\end{tabular}

tion between autonomic functionality and CV mortality is documented [20]. Increased HRV reflects a healthy ANS that is able to respond to changes in the environmental circumstances. By contrast, decreased HRV is a marker of ANS inflexibility, which may precede more sys- temic problems [21].

Depressed HRV has been reported in several CVD, including coronary artery disease (CAD) and heart failure. Actually, HRV is a strong and independent predictor of mortality in CAD patients (after MI). HRV is depressed in these patients, with a reduction in the total power of the signal, presenting some parameters that indicate a prevalence of sympathetic activation, which may lead to cardiac electrical instability. Thus, HRV parameters (time domain, frequency domain) should be explored as quantitative markers of ANS activity, as they are significantly correlated with all-cause mortality, cardiac death, and arrhythmic death [20,22].

HRV is usually assessed with two types of recordings:
1) short-term (e.g. 5 minutes); 2) long-term ( 24 hours). Although the latter is a stronger risk predictor, HRV assessed from short recordings also provides useful prognostic information [20]. Ideally, HRV parameters should be assessed within one week after MI. However these parameters are significant mortality predictors even when measured after that period [23].

Current risk assessment tools do not include HRV parameters. However, there are several HRV derived parameters that can potentially be applied to improve the CVD risk assessment. The flexibility of the developed Bayesian global model solves this problem, as it allows a straightforward integration of additional knowledge/new risk factors.

This is the main focus of the ongoing research: the selection and incorporation of HRV parameters in order to improve risk assessment and consequently the patients' stratification. The incorporation mechanism is assured by the developed combination methodology however the 
selection of the specific HRV parameters must be carefully considered.

Time domain parameters may include: 1) HRV mean, the average value of RR interbeat intervals; 2) HRV SDNN the normal-to-normal (NN) intervals standard deviation; 3) $H R V R M S S D$ the square root of the mean squared differences of successive NN intervals; 4) HRV NN50 the number of interval differences of successive $\mathrm{NN}$ intervals greater than $50 \mathrm{~ms}$; and 5) $H R V$ pNN50 the proportion of NN50 considering the total of NN intervals.

In the frequency domain, three main spectral components can be identified: the very low frequency (VLF: $0.01-0.04 \mathrm{~Hz}$ ), the low frequency (LF: $0.04-0.15 \mathrm{~Hz}$ ) and the high frequency components (HF: $0.15-0.4 \mathrm{~Hz}$ ). Changes in the LF and the HF components reflect sympathetic and parasympathetic activities, and their ratio $(\mathrm{LF} / \mathrm{HF})$ is considered as a marker of the sympatho-vagal balance controlling the heart rate [22]. A different energy distribution was observed in MI patients, VLF components are responsible for the main amount while a minor part is assigned to HF components [23]. The correlation between these components and specific conditions must be further investigated to obtain the required data to perform the incorporation in the global framework. Spectral analysis must be conducted on the HRV signals obtained from the ECG recordings performed on CAD patients.

Non-linear phenomena are also involved in HRV as cardiac activity is also regulated by intrinsically nonlinear mechanisms. Some non-linear parameters can be identified such as $1 / \mathrm{f}$ slope of Fourier spectra, Sample Entropy, Lempel-Ziv Complexity.

The impact of HRV parameters in the characterization of CAD patients will be conducted during hospitalization of these patients in LPHC. An integrated clinical platform, integrating the developed algorithms, will be implemented. In addition to the information obtained from the hospital information system, ECG (Holter) signals will be collected to derive the HRV parameters.

\section{Conclusion}

The two developed methodologies improved the performance of risk assessment when compared to the one achieved by the current risk assessment tools. Moreover, the combination methodology allows important features such as the ability to deal with missing risk factors as well as the incorporation of new risk factors. However, we believe that the incorporation of the Heart Rate Variability parameters can significantly improve the risk assessment/patient stratification.

\section{Acknowledgements}

This work was partially financed by iCIS (CENTRO-07ST24-FEDER-002003) and by Cardiorisk (PTDC/EEI-SII/
2002/2012).

\section{REFERENCES}

[1] WHO, World Health Organization "Cardiovascular Diseases," Fact Sheet No. 317, 2009. http://www.who.int/mediacentre

[2] EHN, European Heart Network, "Healthy Hearts for All," Annual Report 2009, 2010.

http://www.ehnheart.org/publications/annual-reports.html

[3] H. Reiter, et al., "HeartCycle: Compliance and Effectiveness in HF and CAD Closed-Loop Management," Proceedings of the 31st Conference of the IEEE EMBS, 2009.

[4] N. Boye, et al., "PREVE White Paper-ICT Research Directions in Disease Prevention,” 2010.

[5] I. Graham, et al., "Guidelines on Preventing Cardiovascular Disease in Clinical Practice: Executive Summary,” European Heart Journal, Vol. 28, 2007, pp. 2375-2414.

[6] J. Perk, et al., "European Guidelines on Cardiovascular Disease Prevention in Clinical Practice," European Heart Journal, Vol. 33, 2012, pp. 1635-1701.

[7] NVDPA, "Guidelines for the Assessment of Absolute Cardiovascular Disease Risk,” National Heart Foundation of Australia, 2009.

[8] E. Antman, et al., "The TIMI Risk Score for Unstable Angina/Non-St Elevation MI-A Method for Prognostication and Herapeutic Decision Making," Journal of American Medical Association-JAMA, Vol. 284, No. 7, 2000, pp. 835-842.

[9] E. Boersma, et al., "Predictors of Outcome in Patients with Acute Coronary Syndromes without Persistent ST-Segment Elevation; Results from an International Trial of 9461 Patients," Circulation, American Heart Association-AHA, Vol. 101, 2000, pp. 2557-2657.

[10] E. Tang, C. Wong and P. Herbinson, "Global Registry of Acute Coronary Events (GRACE) Hospital Discharge Risk Scores Accurately Predicts Long Term Mortality Post-Acute Coronary Syndrome," American Heart Journal, Vol. 153, No. 1, 2007, pp. 30-35.

[11] E. Auer and R. Kohavi, “An Empirical Comparison of Voting Classification Algorithms: Bagging, Boosting and Variants,” Machine Learning, Vol. 36, 1998, pp. 1-38.

[12] Tsymbal, et al., "Ensemble Feature Selection with the Simple Bayesian Classification,” Information Fusion, Vol. 4, No. 2, 2003, pp. 87-100.

[13] G. Samsa, G. Hu and M. Root, "Combining Information from Multiple Data Sources to Create Multivariable Risk Models: Illustration and Preliminary Assessment of a New Method,” Journal of Biomedical Biotechnology, Vol. 2, 2005, pp. 113-123.

[14] Twardy, "Knowledge Engineering Cardiovascular Bayesian Networks from the Literature,” Technical Report 2005/170, Monash University, 2005.

[15] P. Gonçalves, et al., "TIMI, Pursuit and Grace Risk Scores: Sustained Prognostic Value and Interaction with Revascularization in NSTE-ACS," European Heart Journal, Vol. 26, 2005, pp. 865-872. 
http://dx.doi.org/10.1093/eurheartj/ehi187

[16] S. Paredes, T. Rocha, P. de Carvalho, J. Henriques, J. Morais, J. Ferreira and M. Mendes, "Cardiovascular Event Risk Assessment-Fusion of Individual Risk Assessment Tools Applied to the Portuguese Population," Proceedings of the 15th International Conference on Information Fusion, 2012, pp. 925-932.

[17] S. Paredes, T. Rocha, P. de Carvalho, J. Henriques, J. Morais, J. Ferreira and M. Mendes, "Improvement of CVD Risk Assessment Tools' Performance through Innovative Patients' Grouping Strategies,” Proceedings of the 34th Annual International Conference of the IEEE Engineering in Medicine and Biology Society, 2012.

[18] N. Friedman, et al., "Bayesian Network Classifiers," Machine Learning, Vol. 29, 1997, pp. 131-163.

[19] J. Han, M. Kamber and J. Pei, "Data Mining: Concepts and Techniques,” 3rd Edition, Morgan Kaufmann, 2011.

[20] "Heart Rate Variability. Standards of Measurement, Physiological Interpretation and Clinical Use. Task Force of the European Society of Cardiology and the North Amer- ican Society of Pacing and Electrophysiology,” European Heart Journal, Vol. 17, 1996, pp. 354-381.

http://dx.doi.org/10.1093/oxfordjournals.eurheartj.a01486 8

[21] A. Kemp, et al., "Depression, Comorbid Anxiety Disorders, and Heart Rate Variability in Physically Healthy, Unmedicated Patients: Implications for Cardiovascular Risk,” PLoS One, Vol. 7, No. 2, 2012. http://dx.doi.org/10.1371/journal.pone.0030777

[22] M. Pagani, et al., "Power Spectral Analysis of Heart Rate and Arterial Pressure Variabilities as a Marker of Sympatho-Vagal Interaction in Man and Conscious Dog," Circulation, Vol. 59, 1986, pp. 178-193. http://dx.doi.org/10.1161/01.RES.59.2.178

[23] J. Bigger, et al., "Frequency Domain Measures of Heart Period Variability to Assess Risk Late after Myocardial Infarction," Journal of the American College of Cardiology, Vol. 21, 1993, pp. 729-736. http://dx.doi.org/10.1016/0735-1097(93)90106-B 